\title{
YANG-BAXTER MAPS AND THE DISCRETE KP HIERARCHY
}

\author{
S. KAKEI \\ Department of Mathematics, Rikkyo University, Nishi-ikebukuro, Toshima-ku, Tokyo 171-8501, Japan \\ e-mail:kakei@rkmath.rikkyo.ac.jp \\ J. J. C. NIMMO \\ Department of Mathematics, University of Glasgow, Glasgow G128QQ, UK \\ e-mail:jjcn@maths.gla.ac.uk \\ and R. WILLOX \\ Graduate School of Mathematical Sciences, University of Tokyo, 3-8-1 Komaba, Meguro-ku, \\ Tokyo 153-8914, Japan \\ e-mail:willox@ms.u-tokyo.ac.jp
}

\begin{abstract}
We present a systematic construction of the discrete KP hierarchy in terms of Sato-Wilson-type shift operators. Reductions of the equations in this hierarchy to 1+1-dimensional integrable lattice systems are considered, and the problems that arise with regard to the symmetry algebra underlying the reduced systems as well as the ultradiscretizability of these systems are discussed. A scheme for constructing ultradiscretizable reductions that give rise to Yang-Baxter maps is explained in two explicit examples.
\end{abstract}

2000 Mathematics Subject Classification. 39A10, 39A12.

1. Introduction. A problem widely recognized as one of the most fundamental open problems in the theory of integrable systems is that of the relation between classical and quantum integrable systems with infinitely many degrees of freedom. Recently a possible route emerged through which a firm connection between the classical and quantum settings might be established: the ultradiscretization of (classical) discrete integrable systems $[\mathbf{8}, \mathbf{2 2}]$. Crystal bases, arising in the zero-temperature limit of quantum enveloping algebras, have been shown to play an important rôle in the description of the dynamical properties of so-called box and ball systems (BBSs) [20], which in turn can be obtained from 1+1-dimensional discrete integrable systems through a special limiting procedure: the ultradiscrete limit [22]. On the other hand, geometric crystals [1] are classical analogues of such crystals. These are such that (quantum) crystal bases can be obtained from them through a limiting procedure very much like the ultradiscrete limit. Geometric crystals have been studied extensively in connection with the combinatorial properties of BBSs [10, 11], but, most importantly, they also offer prime examples of Yang-Baxter (YB) maps [7], i.e. of set-theoretical solutions to the YB equation $[\mathbf{6}, \mathbf{2 6}$. In particular, it can be shown that the $R$-matrix associated to such a YB map (or with the tropical version thereof, obtained from the geometric crystal) in the ultradiscrete limit turns into the combinatorial $R$-matrix that governs the time evolution of the BBS [8]. This $R$-matrix, in turn, is directly related to the crystal associated with that BBS. The question that remains however, 
is how these $R$-matrices are related to the time evolutions described by the (classical) discrete integrable systems, in particular discrete soliton systems, that allow for these particular ultradiscrete limits. An especially interesting problem is to understand how it is exactly that geometric crystals arise in this context, i.e. how the algebraic structure of discrete 1+1-dimensional integrable systems gives rise to that of a geometric crystal. Furthermore, one would like to be able to describe this relationship in terms of the fundamental symmetries of the discrete system, preferably in a general framework in order to cover as many examples as possible.

In this paper we shall describe a rather general method for constructing ultradiscretizable YB maps from 1+1-dimensional discrete (integrable) lattice equations. The general framework which allows us to do so is that of the discrete KP hierarchy. We therefore start by giving an explicit construction of this hierarchy and its associated linear problem, in terms of Sato-Wilson-type shift operators. We then discuss the problem of the reduction of the integrable lattice equations that make up this hierarchy to $1+1$-dimensional ones, such that the underlying symmetry algebra of these lattices is of a given type. In the last section of this paper we describe the procedure that allows us to express the dynamics of such reductions as YB maps. Different types of reductions, related to the same symmetry algebra but giving rise to different YB maps, will be discussed.

2. The discrete KP hierarchy. Over the years there have been several descriptions of hierarchies of higher order lattice equations that, for the right set of continuous variables, have the equations of the KP hierarchy as their continuum limits. The 'standard' approach [5] would rely on introducing discrete variables-through a Miwatype transformation [12] - in the KP bilinear identity, the expansion of which then yields lattice equations of an arbitrarily high order. An explicit determinantal form of the hierarchy thus obtained was given in [17]. A different but not entirely unrelated approach was introduced in $[\mathbf{1 4}, \mathbf{1 5}]$, where such higher order lattice equations were obtained as non-linear relations that the solutions of certain linear integral equations have to satisfy. Our definition of the discrete KP hierarchy and its associated linear problem relies instead on an appropriate discretization of the original Sato approach to the continuous KP hierarchy. Similar attempts can be found in [2] and [23], but the present one is probably the first to exploit two different sets of Sato-Wilson shift operators, in close analogy to the case of the continuous Toda hierarchy [25].

We define the following Sato-Wilson-type operators:

$$
\left.\begin{array}{l}
W(\ell, \mathbf{m}):=I+w_{1} T_{\ell}^{-1}+w_{2} T_{\ell}^{-2}+w_{3} T_{\ell}^{-3}+\cdots, \\
\bar{W}(\ell, \mathbf{m}):=\bar{w}_{0}+\bar{w}_{1} T_{\ell}+\bar{w}_{2} T_{\ell}^{2}+\bar{w}_{3} T_{\ell}^{3}+\cdots
\end{array}\right\}
$$

in terms of functions $w_{i}(\ell, \mathbf{m}), \bar{w}_{i}(\ell, \mathbf{m}), \overline{w_{0}} \neq 0$ which, for the time being, only depend on $M+1$-independent variables $(M \in \mathbb{N}),(\ell, \mathbf{m})=\left(\ell, m_{1}, \ldots, m_{M}\right)$; the $M \rightarrow \infty$ limit will be discussed later on. The $T_{\ell}$ operators are shift operators acting on the $\ell$ variable, $T_{\ell} f(\ell, \mathbf{m})=f(\ell+1, \mathbf{m})$, and we define shift operators $T_{m_{j}}$ acting on the $m_{j}$ variable $(j=1, \ldots, M)$ analogously; $I$ stands for the identity (shift) operator. We also define the projection of operators (1) onto their non-negative part as

$$
A\left(T_{\ell}\right)=\sum_{n \in \mathbb{Z}} a_{n} T_{\ell}^{n}, \quad\left[A\left(T_{\ell}\right)\right]_{\geq 0}:=\sum_{n \geq 0} a_{n} T_{\ell}^{n} .
$$


We then fix the dynamics of the coefficients $w_{i}(\ell, \mathbf{m})$ and $\bar{w}_{i}(\ell, \mathbf{m})$ with respect to the $m_{j}$ variables, by imposing the discrete Sato equations $\left({ }^{\forall} j=1 \cdots M\right)$

$$
\begin{aligned}
& \left(T_{m_{j}} W\right)\left(1-\alpha_{j}+\alpha_{j} T_{\ell}\right)=B_{j} W \\
& \left(T_{m_{j}} \bar{W}\right)\left(1-\alpha_{j}+\alpha_{j} T_{\ell}\right)=B_{j} \bar{W} .
\end{aligned}
$$

In these equations, $\alpha_{j}$ are arbitrary constants, and the shift operators $B_{j}$ are defined as

$$
B_{j}:=\left[\left(T_{m_{j}} W\right)\left(1-\alpha_{j}+\alpha_{j} T_{\ell}\right) W^{-1}\right]_{\geq 0} \equiv \alpha_{j} T_{\ell}+\left(1-\alpha_{j}\right) u_{j},
$$

in terms of a new set of dependent variables:

$$
u_{j}=\frac{T_{m_{j}} \bar{w}_{0}}{\bar{w}_{0}} .
$$

It should be remarked that were it not for the introduction of the second set of SatoWilson operators $\bar{W}$, it would be impossible to determine the constant term (w.r.t. $T_{\ell}$ ) in (4) in terms of a single, well-defined variable. Conversely, the order of the operators $B_{j}$ is fixed because of equation (3) for $W$. Moreover, it is easily seen that the lowest order term in $\bar{W}, \bar{w}_{0}$, has a natural expression in terms of Casorati determinants of arbitrary size $(n)$,

$$
\bar{w}_{0}=\frac{T_{\ell} \tau}{\tau}, \quad \tau(\ell, \mathbf{m})=\left|\begin{array}{ccc}
\phi^{(1)} & \cdots & \Delta_{\ell}^{n-1} \phi^{(1)} \\
\vdots & \ddots & \vdots \\
\phi^{(n)} & \cdots & \Delta_{\ell}^{n-1} \phi^{(n)}
\end{array}\right|,
$$

defined in terms of functions $\phi^{(j)}$ that are only required to satisfy the linear equations

$$
\Delta_{\ell} \phi=\Delta_{m_{k}} \phi
$$

for all $k$. The difference operators $\Delta$ used in these relations are defined as $\Delta_{\ell}:=$ $a\left(T_{\ell}-1\right)$ and $\Delta_{m_{j}}:=b_{j}\left(T_{m_{j}}-1\right)$, for some lattice parameters $a, b_{j} \neq 0$, related to the constants $\alpha_{j}$ in the Sato equations (3) by $\alpha_{j}=a / b_{j}$. Note that the dispersion relations (7) ensure that the tau functions $\tau(\ell, \mathbf{m})$ defined in (6) remain invariant if one changes the difference operators $\Delta_{\ell}$ in their entries to any of the $\Delta_{m_{j}}$ operators. Elementary solutions to (7), depending on an arbitrary parameter $\lambda$, take the form

$$
\varphi_{\lambda}:=\left(1-\frac{\lambda}{a}\right)^{\ell} \prod_{j=1}^{M}\left(1-\frac{\lambda}{b_{j}}\right)^{m_{j}}
$$

and can be used to define two sets of wave functions by means of the formal expansions

$$
\begin{aligned}
\Psi_{\lambda}(\ell, \mathbf{m}) & :=W(\ell, \mathbf{m}) \varphi_{\lambda}=\left[1+\sum_{k=1}^{+\infty} w_{k}\left(1-\frac{\lambda}{a}\right)^{-k}\right] \varphi_{\lambda}, \\
\bar{\Psi}_{\lambda}(\ell, \mathbf{m}) & :=\bar{W}(\ell, \mathbf{m}) \varphi_{\lambda}=\left[\bar{w}_{0}+\sum_{k=1}^{+\infty} \bar{w}_{k}\left(1-\frac{\lambda}{a}\right)^{k}\right] \varphi_{\lambda} .
\end{aligned}
$$


As a direct consequence of the Sato equations (3), these wave functions satisfy linear equations of the type $T_{m_{j}} \Phi=B_{j} \Phi$, or equivalently,

$$
\Phi=\frac{1}{a-b_{j}} \frac{1}{u_{j}}\left[a\left(T_{\ell} \Phi\right)-b_{j}\left(T_{m_{j}} \Phi\right)\right] \quad{ }^{\forall} j=1 \cdots M,
$$

with $u_{j}$ given by

$$
u_{j}=\frac{\tau\left(T_{\ell} T_{m_{j}} \tau\right)}{\left(T_{\ell} \tau\right)\left(T_{m_{j}} \tau\right)} .
$$

The compatibility conditions of the linear system (10), $\left(T_{m_{i}} B_{j}\right) B_{i}=\left(T_{m_{j}} B_{i}\right) B_{j}$, take the form of a system of non-linear difference equations $(\forall i, j=1, \ldots, M ; i \neq j$ ),

$$
\left.\begin{array}{l}
u_{i}\left(T_{m_{i}} u_{j}\right)=u_{j}\left(T_{m_{j}} u_{i}\right), \\
\alpha_{i}\left(1-\alpha_{j}\right)\left(T_{m_{i}} u_{j}\right)+\alpha_{j}\left(1-\alpha_{i}\right)\left(T_{\ell} u_{i}\right)=\alpha_{j}\left(1-\alpha_{i}\right)\left(T_{m_{j}} u_{i}\right)+\alpha_{i}\left(1-\alpha_{j}\right)\left(T_{\ell} u_{j}\right),
\end{array}\right\}
$$

the first equation of which is trivially satisfied if one adopts the tau-function representation (11) for the $u_{i}$. This system comprises $M(M-1) / 2$ equations belonging to the discrete KP hierarchy. The tau-function representation (11) allows one to rewrite (12) in a bilinear form,

$$
\left(b_{i}-b_{j}\right)\left(T_{\ell} \tau\right)\left(T_{m_{i}} T_{m_{j}} \tau\right)+\left(b_{j}-a\right)\left(T_{m_{i}} \tau\right)\left(T_{\ell} T_{m_{j}} \tau\right)+\left(a-b_{i}\right)\left(T_{m_{j}} \tau\right)\left(T_{\ell} T_{m_{i}} \tau\right)=0,
$$

which is of course nothing but the Hirota-Miwa (HM) equation [9, 12]. (In fact, the coefficients in the HM equation (13) depend on the choice of a specific gauge for the tau function, i.e. $\tau \rightarrow C^{m_{i} m_{j}} \tau$, which leaves $\bar{w}_{0}(6)$ invariant). It should be noted also that both the HM equation (13) and the tau-function expression (11) for $u_{j}$ are invariant under a simple gauge with $\varphi_{\lambda}: \tau \rightarrow \tau \varphi_{\lambda}$.

One of the most noteworthy features of the dKP hierarchy is the remarkable isotropy of the associated lattice (here, $M+1$-dimensional). If we denote the HM equation involving independent variables $\left(\ell, m_{i}, m_{j}\right)$ as $\mathbf{E}\left(\ell, m_{i}, m_{j}\right)$, the following statements are readily proven:

(i) The equations in $\mathbb{E}=\left\{\mathbf{E}\left(\ell, m_{j}, m_{k}\right) \mid m_{j}, m_{k} \in\left\{m_{1}, \ldots, m_{M}\right\}, m_{j} \neq m_{k}\right\}$ generate all bilinear equations in $\overline{\mathbb{E}}=\left\{\mathbf{E}\left(\ell_{1}, \ell_{2}, \ell_{3}\right) \mid \ell_{1}, \ell_{2}, \ell_{3} \in\{\ell, \mathbf{m}\}, \ell_{1} \neq \ell_{2} \neq \ell_{3}\right\}$.

(ii) The equations in $\mathbb{E}($ or $\overline{\mathbb{E}})$ generate all equations of the discrete KP hierarchy proposed in [17]:

$$
{ }^{\forall} N=3, \ldots, M+1: \quad\left|\begin{array}{cccc}
\tau_{\ell_{1}} \tau_{\widehat{\ell}_{1}} & \tau_{\ell_{2}} \tau_{\ell_{2}} & \cdots & \tau_{\ell_{N}} \tau_{\widehat{\ell}_{N}} \\
1 & 1 & \cdots & 1 \\
a_{1} & a_{2} & \cdots & a_{N} \\
\vdots & \vdots & & \vdots \\
a_{1}^{N-2} & a_{2}^{N-2} & \cdots & a_{N}^{N-2}
\end{array}\right|=0
$$

where $\tau_{\ell_{k}}=T_{\ell_{k}} \tau, \tau_{\widehat{\ell}_{k}}=\left(\prod_{j \neq k} T_{\ell_{j}}\right) \tau$, for $\left(\ell_{1}, \ldots, \ell_{n}\right) \equiv\left(\ell, m_{1}, \ldots, m_{N-1}\right)$ and $a_{j}=a$ when $j=1$ and $a_{j}=b_{j-1}(j \geq 2)$ otherwise. 
These results basically derive from the fact that the linear system (10), taken together with the HM equation (13), is equivalent to the fully symmetric system

$$
\Phi=\frac{1}{a_{i}-a_{j}} \frac{\left(T_{\ell_{i}} \tau\right)\left(T_{\ell_{j}} \tau\right)}{\tau\left(T_{\ell_{i}} T_{\ell_{j}} \tau\right)}\left[a_{i}\left(T_{\ell_{i}} \Phi\right)-a_{j}\left(T_{\ell_{j}} \Phi\right)\right] \quad\left({ }^{\forall} i \neq j\right) .
$$

That is, although the $\ell$ lattice direction was singled out as special in our construction, the actual set of equations $\overline{\mathbb{E}}$ the tau functions for the discrete KP hierarchy must satisfy is fully isotropic. We therefore define the discrete KP hierarchy to be the infinite set of HM equations obtained from $\overline{\mathbb{E}}$ as $M \rightarrow \infty$. Locally, i.e. in three dimensions, this infinite lattice is always governed by the HM equation (14) at $N=3$.

Furthermore, the equations of order $M+1$ in (14) take the form $\sum_{n=1}^{M+1} \frac{\tau_{\ell_{n}} \tau_{\ell_{n}}}{\prod_{k \neq n}\left(a_{n}-a_{k}\right)}=0$, which is equivalent to the (formal) residue formula

$$
\sum_{n=1}^{M+1} \operatorname{Res}_{\lambda=a_{n}}\left[\tau\left(\mathbf{x}-\boldsymbol{\varepsilon}\left[\lambda^{-1}\right]\right) \tau\left(\mathbf{x}^{\prime}+\boldsymbol{\varepsilon}\left[\lambda^{-1}\right]\right) \prod_{k=1}^{M+1}\left(a_{k}-\lambda\right)^{-1}\right]=0,
$$

where $\mathbf{x}:=\sum_{n=1}^{M+1} \ell_{n} \boldsymbol{\varepsilon}\left[a_{n}^{-1}\right]$ for $\boldsymbol{\varepsilon}[\zeta] \equiv\left(\zeta, \frac{\zeta^{2}}{2}, \ldots, \frac{\zeta^{M+1}}{M+1}\right)$ and $\mathbf{x}^{\prime}=\left(\prod_{n=1}^{M+1} T_{\ell_{n}}^{-1}\right) \mathbf{x}$. When $M \rightarrow \infty$, this relation is nothing but the KP bilinear identity

$$
\oint_{\lambda=\infty} \frac{\mathrm{d} \lambda}{2 \pi i} \tau\left(\mathbf{x}-\varepsilon\left[\lambda^{-1}\right]\right) \tau\left(\mathbf{x}^{\prime}+\varepsilon\left[\lambda^{-1}\right]\right) e^{\sum_{n=1}^{+\infty}\left(x_{n}-x_{n}^{\prime}\right) \lambda^{n}}=0
$$

subject to the Miwa transformation [12]

$$
\mathbf{x} \equiv\left(x_{1}, x_{2}, x_{3}, \ldots\right)=\mathbf{x}_{0}+\sum_{n=1}^{\infty} \ell_{n} \varepsilon\left[a_{n}^{-1}\right], \quad \varepsilon\left[\lambda^{-1}\right]=\left(\lambda^{-1}, \frac{\lambda^{-2}}{2}, \ldots, \frac{\lambda^{-n}}{n}, \ldots\right)
$$

and with $\mathbf{x}-\mathbf{x}^{\prime}=\sum_{n=1}^{\infty} \varepsilon\left[a_{n}^{-1}\right]$, as explained in [5]. In that paper, the tau functions satisfying the above bilinear equations are described by means of a representation of the $g l(\infty)$ algebra in terms of free fermions, in complete analogy to the case of the continous KP hierarchy. The algebra $g l(\infty)$ can therefore be regarded the fundamental symmetry algebra underlying the discrete KP hierarchy. In fact, the invariance of the discrete KP hierarchy (and of the HM equation in particular) under Darboux transformations [16] is a prime example of the action of this symmetry algebra. Given a particular solution $\Phi$ to the linear system (15) corresponding to a given tau function $\tau_{\text {vac }}$, we define the Darboux transformation of this tau function as $\tau^{\mathrm{vac}} \rightarrow \tau^{\mathrm{vac}} \Phi$; solutions of the linear system are transformed by the operator $G_{\Phi}:=a\left(T_{\ell}-\frac{T_{\ell} \Phi}{\Phi}\right)$; i.e. if $\psi$ is also a solution of the linear system (15) for $\tau_{\mathrm{vac}}$, then $G_{\Phi} \psi \equiv\left[\left(T_{\ell} \psi\right) \Phi-\psi\left(T_{\ell} \Phi\right)\right] / \Phi$ will be a solution of the linear system (15) corresponding to the new tau function $\tau^{\mathrm{vac}} \Phi$. Iteration of this procedure starting from $\tau^{\mathrm{vac}}=1$, yields Casorati-type tau functions as those in (6). (Note that one recovers the dispersion relations (7) from the linear system (15) if $\tau=1$.)

Furthermore, it is easily seen that a shift in the $\ell$-direction can, itself, be interpreted as a special Darboux transformation: $\left.\tau \rightarrow\left(\tau \bar{\Psi}_{\lambda} \varphi_{\lambda}^{-1}\right)\right|_{\lambda=a} \equiv T_{\ell} \tau$. This offers yet another perspective on the discrete KP hierarchy: one can think of the entire hierarchy as arising from a single copy of the HM equation, augmented with time evolutions 
(in fact symmetries) that correspond to all Darboux transformations of that equation. A final comment concerns the characterization of the tau functions as solutions to a linear integral equation [28]:

$$
\tau\left(\mathbf{x}-\boldsymbol{\varepsilon}\left[k^{-1}\right]\right)=\tau(\mathbf{x})-\int_{\mathcal{C}_{\lambda}} \frac{\mathrm{d} \lambda}{2 \pi i} \int_{\mathcal{C}_{\mu}} \frac{\mathrm{d} \mu}{2 \pi i} h(\lambda, \mu) \frac{e^{\sum_{n=1}^{+\infty} x_{n}\left(\lambda^{n}-\mu^{n}\right)}}{k-\mu} \tau\left(\mathbf{x}-\boldsymbol{\varepsilon}\left[\lambda^{-1}\right]\right) .
$$

For a given kernel $h(\lambda, \mu)$ and for $\mathbf{x}$ as defined above, any $\tau(\mathbf{x})$ satisfying (19) will be a tau function for the discrete KP hierarchy. This offers a direct link to the construction found in [13], where it was shown that such linear integral equations can be used to generate linear problems and Bäcklund transformations for integrable lattice equations, including the discrete KP lattice and several of its 1+1-dimensional reductions.

3. Reductions of the discrete KP hierarchy. Soliton solutions for the discrete KP hierarchy can be expressed in the form of Casorati determinants (6), with entries that are appropriate linear combinations of the elementary solutions $\varphi_{\lambda}$ to the vacuum linear problem (15) for $\tau=1$. In particular, defining

$$
\varphi_{p, q}:=\prod_{n=1}^{\infty}\left(\frac{a_{n}-q}{a_{n}-p}\right)^{\ell_{n}} \quad p, q \in \mathbb{C}
$$

one has 1- and 2-soliton solutions

$$
\begin{gathered}
\tau_{1-\mathrm{sol}}=1+\varphi_{p, q}, \\
\tau_{2-\mathrm{sol}}=1+\varphi_{p_{1}, q_{1}}+\varphi_{p_{2}, q_{2}}+\frac{\left(p_{1}-p_{2}\right)\left(q_{1}-q_{2}\right)}{\left(p_{1}-q_{2}\right)\left(q_{1}-p_{2}\right)} \varphi_{p_{1}, q_{1}} \varphi_{p_{2}, q_{2}} .
\end{gathered}
$$

Under the Miwa transformation (18) and at the limit $a_{n} \rightarrow \infty$, the functions $\varphi_{p, q}$ tend to $\exp \sum_{n=1}^{\infty} x_{n}\left(p^{n}-q^{n}\right)$, and the soliton solutions tend to those of the continuous KP hierarchy. More precisely, due to the Miwa transformation (18), for $\left|a_{n}\right| \gg 1$ one can write

$$
T_{\ell_{n}} \tau=\sum_{j=0}^{\infty} a_{n}^{-j}\left[p_{j}(\tilde{\partial}) \tau(\mathbf{x})\right]
$$

where $p_{j}(\tilde{\partial})$ denotes for the Schur polynomial of weight $j, p_{j}\left(\partial_{x_{1}}, \frac{1}{2} \partial_{x_{2}}, \ldots, \frac{1}{n} \partial_{x_{n}}, \ldots\right)$.

It is worth emphasizing that when considering dimensional reductions of the discrete KP hierarchy, one should study both the behaviour of the equations under this natural continuum limit as well as that of their solutions. For example, the simplest possible, non-trivial reduction is the one in which the tau functions that solve the HM equation (13) have to satisfy the constraint $T_{m} T_{n} \tau=\tau$ (where for simplicity we have chosen $m$ and $n$ to represent the two distinct directions $m_{i}$ and $\left.m_{j}\right)$. In this case the HM equation $\mathbf{E}(\ell, m, n)$ reduces to

$$
(1-\delta) \tau\left(T_{\ell} \tau\right)+\delta\left(T_{m} \tau\right)\left(T_{\ell} T_{m}^{-1} \tau\right)=\left(T_{\ell} T_{m} \tau\right)\left(T_{m}^{-1} \tau\right)
$$

with $\delta=\frac{a-c}{a-b}\left(b \equiv b_{i}, c \equiv b_{j}\right)$, and the corresponding discrete KP equation (12) yields

$$
\frac{1}{\left(T_{\ell} T_{m} u\right)}-\frac{1}{u}=\delta\left[\left(T_{\ell} u\right)-\left(T_{m} u\right)\right]
$$


in the variable $u=\frac{\tau\left(T_{\ell} T_{m}^{-1} \tau\right)}{\left(T_{\ell} \tau\right)\left(T_{m}^{-1} \tau\right)}$. This equation is often referred to as the discrete $\mathrm{KdV}(\mathrm{dKdV})$ equation, as consecutive continuum limits $a \rightarrow \infty, b \rightarrow \infty$ indeed yield the $\mathrm{KdV}$ equation after an appropriate change of (dependent) variables: $u=1-U /(2 a b)$. In addition to the constraint on the tau functions, one can also impose the following reduction condition on the solutions of the linear problem (15): $T_{m} T_{n} \psi=(1-\lambda / b)(1-\lambda / c) \psi$, for some spectral parameter $\lambda$. The resulting linear system is

$$
\begin{aligned}
& {\left[T_{\ell}^{2}+\left[\left(\frac{b}{a}-1\right) \frac{1}{T_{\ell} T_{m} u}+\left(\frac{c}{a}-1\right)\left(T_{m} u\right)\right] T_{\ell}\right] \psi=\frac{(\lambda-a)(\lambda+a-b-c)}{a^{2}} \psi,} \\
& T_{m} \psi=\left[\frac{a}{b} T_{\ell}+\left(1-\frac{a}{b}\right) \frac{1}{T_{m} u}\right] \psi,
\end{aligned}
$$

which yields the KdV Lax pair in the above continuum limit. However, if one takes a closer look at the behaviour of the constraint $T_{m} T_{n} \tau=\tau$ at the continuum limit, it is immediately clear that extra care is needed if one wishes to obtain non-trivial limits for the solutions to the discrete equation (24). Substituting the expansion (22) in the reduction constraint, one obtains

$$
\tau=\tau+\left(\frac{1}{b}+\frac{1}{c}\right) \tau_{x_{1}}+\frac{1}{2}\left(\frac{1}{b^{2}}+\frac{1}{c^{2}}\right) \tau_{x_{2}}+\frac{1}{2}\left(\frac{1}{b}+\frac{1}{c}\right)^{2} \tau_{2 x_{1}}+\mathcal{O}(3)
$$

i.e. unless $b+c=0$, the tau functions for the continuous version of the reduced equation (24) will not depend on the lowest weight variable $x_{1}$ and thus will all be trivial. If on the contrary, one imposes the condition $b+c$ on the solutions of (24), the reduction condition on the continuous tau functions obtained from (26) is $\tau_{x_{2}}=0$, exactly as one would expect when reducing the continuous KP equation to the KdV equation. Furthermore, for the soliton solutions to satisfy the reduction constraint, it is necessary and sufficient for the functions $\varphi_{p, q}$ to be invariant under combined shifts: $T_{m} T_{n} \varphi_{p, q}=\varphi_{p, q}$. This yields a condition on the spectral parameters $p$ and $q, p+q=b+c$, which under the previous assumptions yields $p=-q$. This is of course nothing but the reduction condition required to reduce $g l(\infty)$ to the affine Lie algebra $A_{1}^{(1)}$, i.e. to the symmetry algebra underlying the KdV hierarchy (cf. [4] for a detailed discussion of such reductions). If of course, one chooses to study the discrete lattices obtained through such reductions for their own sake, i.e. if one admits the possibility that their solutions might only have trivial continuum limits, it might seem there is no compelling reason to impose any restrictions on the lattice parameters themselves, were it not for the problems the algebraic interpretation of such reductions pose. Reduction conditions (on the soliton solutions of the KP equation) of the type $p^{n}-q^{n}=\mathrm{c}(p-q), p \neq q$ for some non-zero constant $c$ are referred to as 'pseudoreductions', and their geometric interpretation has been clarified in [19]: the integrable equations found in a pseudo-reduced KP hierarchy are mere recombinations of the flows in the hierarchy one would obtain if the constant that appears in the reduction condition were exactly equal to zero (hence, in this case, mere recombinations of $\mathrm{KdV}$ and its higher order flows). However, in the discrete case there is no such clear-cut answer, and the question of what the underlying symmetry algebras of such 'pseudoreduced' discrete KP hierarchies are remains an intriguing problem. 
Another interesting problem is that of the classification of possible reductions of the discrete KP hierarchy, according to their corresponding reduction constraints. Imposing the above KdV-type constraint on the Sato-Wilson operator $W$, we obviously have

$$
W\left(\ell, m_{1}+1, m_{2}+1\right)=W\left(\ell, m_{1}, m_{2}\right)
$$

where we only indicate those independent variables that are used in the reduction and/or the reduced system (equation (24) was expressed in terms of $\ell$ and $m_{1}$ ). Note also that under this condition, the corresponding wave functions (9) automatically satisfy (25). However, retaining the same independent variables $\ell$ and $m_{1}$, there is a second possible reduction condition,

$$
W\left(\ell, m_{1}, m_{2}+1, m_{3}+1\right)=W\left(\ell, m_{1}, m_{2}, m_{3}\right),
$$

which now involves a fourth direction on the original discrete KP lattice. Under the above assumptions, both reductions clearly correspond to the same symmetry algebra, $A_{1}^{(1)}$, but as we shall see in the next section, they describe quite different dynamics.

We shall also briefly discuss the three possible reductions involving three shifts that result in reduced lattices expressible in terms of the $\ell$ and $m_{1}$ variables and which are related to the $A_{2}^{(1)}$ algebra:

$$
\begin{array}{rlrl}
\text { (i) } & W\left(\ell+1, m_{1}+1, m_{2}+1\right) & =W\left(\ell, m_{1}, m_{2}\right), \\
\text { (ii) } & W\left(\ell, m_{1}+1, m_{2}+1, m_{3}+1\right) & =W\left(\ell, m_{1}, m_{2}, m_{3}\right), \\
& \text { (iii) } & W\left(\ell, m_{1}, m_{2}+1, m_{3}+1, m_{4}+1\right) & =W\left(\ell, m_{1}, m_{2}, m_{3}, m_{4}\right) .
\end{array}
$$

4. BBSs and YB maps. The classical BBs due to Takahashi and Satsuma [21]

$$
U_{\ell}^{t+1}=\min \left[1-U_{\ell}^{t}, \sum_{j=\infty}^{\ell-1}\left(U_{j}^{t}-U_{j}^{t+1}\right)\right],
$$

where $U_{\ell}^{t} \in\{0,1\}$ and with boundary conditions $U_{\ell}^{t} \equiv 0$ when $|\ell| \gg 1$, is known to be related to the $\mathrm{dKdV}$ equation through ultradiscretization $[\mathbf{2 2}, \mathbf{2 4}]$. This procedure relies on the positivity of the (dependent) variables and parameters of a given lattice equation and involves introducing a small positive parameter $(\epsilon)$ in the solutions of these equations, which are then subjected to a limiting procedure. For example, rewriting the $\mathrm{dKdV}$ equation (24) as

$$
\frac{1}{\left(T_{\ell} T_{m} u\right)}+\delta\left(T_{m} u\right)=\frac{1}{u}+\delta\left(T_{\ell} u\right)
$$

and assuming positivity of the solutions $u(\ell, m)$ and $\delta$, one can introduce a small $\epsilon$ dependence by writing $u=\exp U / \epsilon, \delta=\exp \Delta / \epsilon$. Taking the limit

$$
\lim _{\epsilon \rightarrow 0^{+}} \epsilon \log \left(e^{\frac{-\left(T_{\ell} T_{m} U\right)}{\epsilon}}+e^{\frac{\Delta+\left(T_{m} U\right)}{\epsilon}}\right)=\lim _{\epsilon \rightarrow 0^{+}} \epsilon \log \left(e^{\frac{-U}{\epsilon}}+e^{\frac{\Delta+\left(T_{\ell} U\right)}{\epsilon}}\right),
$$

of (33) one obtains $\max \left[-\left(T_{\ell} T_{m} U\right),\left(T_{m} U\right)+\Delta\right]=\max \left[-U,\left(T_{\ell} U\right)+\Delta\right]$. Unfortunately, since the max operation is present on both its left- and right-hand sides, this relation does not yield an evolution equation for the variable $U(\ell, m)$, and hence the 
$\mathrm{dKdV}$ equation is not 'ultradiscretizable' in this naive sense. In fact, a rather clever separation of variables is needed to overcome this problem [24]. Here we wish to propose a systematic procedure for constructing ultradiscretizable 1+1-dimensional integrable lattices. First, we implement the constraint which led to the linear problem for the $\mathrm{dKdV}$ equation (27), $\Psi_{\zeta}\left(\ell, m_{1}+1, m_{2}+1\right)=\zeta \Psi_{\zeta}\left(\ell, m_{1}, m_{2}\right)$, by means of the auxiliary variable $\Phi_{\zeta}\left(\ell, m_{1}, m_{2}\right):=\Psi_{\zeta}\left(\ell, m_{1}, m_{2}+1\right)$. We thus obtain the $2 \times 2$ linear problem

$$
\begin{aligned}
T_{\ell}\left[\begin{array}{l}
\Psi_{\zeta} \\
\Phi_{\zeta}
\end{array}\right] & =\left[\begin{array}{cc}
\left(1-\alpha_{2}^{-1}\right) u & \alpha_{2}^{-1} \\
\alpha_{1}^{-1} \zeta & \left(1-\alpha_{1}^{-1}\right) u^{-1}
\end{array}\right]\left[\begin{array}{l}
\Psi_{\zeta} \\
\Phi_{\zeta}
\end{array}\right], \\
T_{m_{1}}\left[\begin{array}{c}
\Psi_{\zeta} \\
\Phi_{\zeta}
\end{array}\right] & =\left[\begin{array}{cc}
\left(1-\alpha_{1}\right) v^{-1} & \alpha_{1} \alpha_{2}^{-1} \\
\zeta & 0
\end{array}\right]\left[\begin{array}{l}
\Psi_{\zeta} \\
\Phi_{\zeta}
\end{array}\right],
\end{aligned}
$$

where $u$ is defined as before and $v^{-1}:=\frac{1}{T_{m_{1}} u}-\frac{\alpha_{1}\left(1-\alpha_{2}\right)}{\alpha_{2}\left(1-\alpha_{1}\right)} u$. The compatibility condition of this sytem is

$$
T_{m_{1}} u=\frac{v}{1+\delta u v}, \quad T_{\ell} v=u(1+\delta u v)
$$

with $\delta$ as before. This system obviously implies that $u=\delta^{-1}\left(\frac{1}{T_{m_{1}} u}-\frac{1}{v}\right)$ and $T_{\ell} v=\frac{u v}{T_{m_{1}} u}$, which after a change of variables $m_{1}=-t$ (i.e. $T_{m_{1}} \equiv T_{t}^{-1}$ ) can be rewritten as

$$
u_{\ell}^{t+1}=\frac{1}{\delta}\left(\frac{1}{u_{\ell}^{t}}-\frac{1}{v_{\ell}^{t+1}}\right), \quad v_{\ell}^{t+1}=\frac{u_{\ell-1}^{t+1}}{u_{\ell-1}^{t}} v_{\ell-1}^{t+1} .
$$

The last equation in this system can be solved explicitly for the boundary conditions $u_{\ell}^{t}=1$ and $v_{\ell}^{t}=\frac{1}{1-\delta}$ for $\ell \ll 0$, yielding $v_{\ell}^{t+1} \equiv \frac{1}{1-\delta} \prod_{j=-\infty}^{\ell-1} \frac{u_{j}^{t+1}}{u_{j}^{t}}$ and, most importantly,

$$
u_{\ell}^{t+1}=\frac{1}{\delta} \frac{1}{u_{\ell}^{t}}+\left(1-\frac{1}{\delta}\right) \prod_{j=-\infty}^{\ell-1} \frac{u_{j}^{t}}{u_{j}^{t+1}} .
$$

Assuming overall positivity, one can put $u_{\ell}^{t}=\exp \left(-U_{\ell}^{t} / \epsilon\right), \delta=\exp (1 / \epsilon)>1$, upon which the ultradiscrete limit of (38) yields $U_{\ell}^{t+1}=-\max \left[U_{\ell}^{t}-1, \sum_{j=-\infty}^{\ell-1}\left(U_{j}^{t+1}-U_{j}^{t}\right)\right]$ which is equivalent to the BBS (32).

This alternative form (36) of the $\mathrm{dKdV}$ lattice is not only crucial for the ultradiscretization to yield a meaningful (evolutive) system, but it also defines a $Y B$ map [26], i.e. a set theoretical solution to the YB equation in the sense of Drinfeld [6]. Following [27] we consider the general, spectral-parameter-dependent YB equation

$$
R_{12}\left(\lambda_{1}, \lambda_{2}\right) R_{13}\left(\lambda_{1}, \lambda_{3}\right) R_{23}\left(\lambda_{2}, \lambda_{3}\right)=R_{23}\left(\lambda_{2}, \lambda_{3}\right) R_{13}\left(\lambda_{1}, \lambda_{3}\right) R_{12}\left(\lambda_{1}, \lambda_{2}\right),
$$

where $R$ is a map from the square of some set $X$ to itself

$$
\begin{array}{ccc}
R: X \times X & \longrightarrow & X \times X \\
\Psi & & \Psi \\
(x, y) & \mapsto & (f(x, y), g(x, y))
\end{array}
$$


and $R_{i j}$ is the corresponding action on $X^{n}$ :

$$
\begin{array}{cccc}
R_{i j}: & X^{n} & X^{n} \\
\Psi & & \Psi \\
& \left(\ldots, x_{i}, \ldots, x_{j}, \ldots\right) & \mapsto & \left(\ldots, f\left(x_{i}, x_{j}\right), \ldots, g\left(x_{i}, x_{j}\right), \ldots\right) .
\end{array}
$$

Solutions of this type to the YB equation are called YB maps and a plethora of examples related to classical integrable systems are known; an excellent review of YB maps, including several examples, can be found in [27].

Inspired by the form of (36), we define the map

$$
R(\delta): \quad(u, v) \mapsto\left(\frac{v}{1+\delta u v}, u(1+\delta u v)\right),
$$

such that the $\mathrm{dKdV}$ evolution takes the form $\left(T_{m_{1}} u, T_{\ell} v\right)=R(\delta)(u, v)$. This map, as it stands, is not a YB map. It does however satisfy a YB equation when thought of as a degenerate version of the map

$$
R(\kappa, \mu): \quad(u, v) \mapsto\left(\frac{v(1+\mu u v)}{1+\kappa u v}, \frac{u(1+\kappa u v)}{1+\mu u v}\right),
$$

which is a 'symmetrized' version of the $\mathrm{dKdV}$ lattice, such that $R(\delta, 0)=R(\delta)$. A straightforward calculation shows that the map (43) satisfies the YB equation (39) and hence that the map $R(\delta)$ itself satisfies

$$
R_{12}\left(\delta_{1}, \delta_{2}\right) R_{13}\left(\delta_{1}\right) R_{23}\left(\delta_{2}\right)=R_{23}\left(\delta_{2}\right) R_{13}\left(\delta_{1}\right) R_{12}\left(\delta_{1}, \delta_{2}\right) .
$$

The map (43) is identical to the Harrison-type map (47) in [18], up to rescaling of the variables. This map is in fact related to the $A_{1}^{(1)}$ reduction of type (28). Using Sato-Wilson operators that satisfy (28) to define wave functions $\Psi_{\zeta}$, we have $\Psi_{\zeta}\left(\ell, m_{1}, m_{2}+1, m_{3}+1\right)=\zeta \Psi_{\zeta}\left(\ell, m_{1}, m_{2}, m_{3}\right)$. Introducing the auxiliary variable $\Phi_{\zeta}\left(\ell, m_{1}, m_{2}, m_{3}\right):=\Psi_{\zeta}\left(\ell, m_{1}, m_{2}+1, m_{3}\right)$ we obtain the $2 \times 2$ linear problem

$$
\begin{aligned}
T_{\ell}\left[\begin{array}{l}
\Psi_{\zeta} \\
\Phi_{\zeta}
\end{array}\right]=\left[\begin{array}{cc}
\left(1-\alpha_{2}^{-1}\right) u & \alpha_{2}^{-1} \\
\alpha_{3}^{-1} \zeta & \left(1-\alpha_{3}^{-1}\right) u^{-1}
\end{array}\right]\left[\begin{array}{l}
\Psi_{\zeta} \\
\Phi_{\zeta}
\end{array}\right], \\
T_{m_{1}}\left[\begin{array}{c}
\Psi_{\zeta} \\
\Phi_{\zeta}
\end{array}\right]=\left[\begin{array}{cc}
\left(1-\alpha_{1} \alpha_{2}^{-1}\right) v^{-1} & \alpha_{1} \alpha_{2}^{-1} \\
\alpha_{1} \alpha_{3}^{-1} \zeta & \left(1-\alpha_{1} \alpha_{3}^{-1}\right) v
\end{array}\right]\left[\begin{array}{c}
\Psi_{\zeta} \\
\Phi_{\zeta}
\end{array}\right]
\end{aligned}
$$

the compatibility condition of which is

$$
T_{m_{1}} u=v \frac{\alpha_{1}\left(1-\alpha_{3}^{-1}\right)+\left(\alpha_{1} \alpha_{3}^{-1}-1\right) u v}{\left(\alpha_{1} \alpha_{2}^{-1}-1\right)+\alpha_{1}\left(1-\alpha_{2}^{-1}\right) u v}, \quad T_{\ell} v=\frac{u v}{T_{m_{1}} u} .
$$

This system is equivalent to the action of the YB map (43),

$$
\left(T_{m_{1}} u, T_{\ell} v\right)=R\left(\frac{\alpha_{3}\left(\alpha_{2}-1\right)}{\alpha_{2}\left(\alpha_{3}-1\right)}, \frac{\alpha_{3}\left(\alpha_{1}-\alpha_{3}\right)\left(\alpha_{1}-\alpha_{2}\right)}{\alpha_{1}^{2} \alpha_{2}\left(\alpha_{3}-1\right)^{2}}\right)(u, v),
$$


up to rescaling of the field $v: v \rightarrow \frac{\alpha_{3}\left(\alpha_{1}-\alpha_{2}\right)}{\alpha_{1} \alpha_{2}\left(\alpha_{3}-1\right)} v$. However, not only is the discrete system obtained from this reduction associated with a YB map, but it is also ultradiscretizable for an appropriate choice of the parameters (so as to ensure positivity). In fact, its ultradiscrete limit can be shown to be equivalent to the BBS with carrier introduced in [20], which can be interpreted as the soliton cellular automaton that corresponds to the crystal obtained from the symmetric tensor representation of $U_{q}^{\prime}\left(A_{1}^{(1)}\right)$ in the limit $q \rightarrow 0[11]$.

The above procedure also generalizes to higher orders. If we consider the reduction (31), the wave functions obtained from such Sato-Wilson operators satisfy $\Psi_{\zeta}\left(\ell, m_{1}, m_{2}+1, m_{3}+1, m_{4}+1\right)=\zeta \Psi_{\zeta}\left(\ell, m_{1}, m_{2}, m_{3}, m_{4}\right)$. Introducing new variables $\Phi_{\zeta}\left(\ell, m_{1}, m_{2}, m_{3}, m_{4}\right):=\Psi_{\zeta}\left(\ell, m_{1}, m_{2}+1, m_{3}, m_{4}\right)$ and $\Xi_{\zeta}\left(\ell, m_{1}, m_{2}, m_{3}, m_{4}\right):=$ $\Psi_{\zeta}\left(\ell, m_{1}, m_{2}+1, m_{3}+1, m_{4}\right)$, we obtain the following $3 \times 3$ linear problem:

$$
\begin{aligned}
& T_{\ell}\left[\begin{array}{c}
\Psi_{\zeta} \\
\Phi_{\zeta} \\
\Xi_{\zeta}
\end{array}\right]=\left[\begin{array}{ccc}
\left(1-\alpha_{2}^{-1}\right) u_{1} & \alpha_{2}^{-1} & 0 \\
0 & \left(1-\alpha_{3}^{-1}\right) u_{2} & \alpha_{3}^{-1} \\
\alpha_{4}^{-1} \zeta & 0 & \left(1-\alpha_{4}^{-1}\right) u_{3}
\end{array}\right]\left[\begin{array}{c}
\Psi_{\zeta} \\
\Phi_{\zeta} \\
\Xi_{\zeta}
\end{array}\right], \\
& T_{m_{1}}\left[\begin{array}{l}
\Psi_{\zeta} \\
\Phi_{\zeta} \\
\Xi_{\zeta}
\end{array}\right]=\left[\begin{array}{ccc}
\left(1-\alpha_{1} \alpha_{2}^{-1}\right) v_{1} & \alpha_{1} \alpha_{2}^{-1} & 0 \\
0 & \left(1-\alpha_{1} \alpha_{3}^{-1}\right) v_{2} & \alpha_{1} \alpha_{3}^{-1} \\
\alpha_{1} \alpha_{4}^{-1} \zeta & 0 & \left(1-\alpha_{1} \alpha_{4}^{-1}\right) v_{3}
\end{array}\right]\left[\begin{array}{c}
\Psi_{\zeta} \\
\Phi_{\zeta} \\
\Xi_{\zeta}
\end{array}\right],
\end{aligned}
$$

for functions $u_{i}, v_{i}$ subject to $u_{1} u_{2} u_{3}=v_{1} v_{2} v_{3}=1$. Further conditions on these variables arise from the compatibility condition of the above linear equations,

$$
T_{m_{1}} u_{j}=\frac{u_{j}\left(\mu_{j+1} u_{j+1}-v_{j+1} v_{j+1}\right)}{\mu_{j} u_{j}-v_{j} v_{j}}, \quad T_{\ell} v_{j}=\frac{v_{j}\left(T_{m_{1}} u_{j}\right)}{u_{j}} \quad j=1,2,3 \text {, }
$$

defined cyclically, $\left(u_{4}, v_{4}, \mu_{4}, v_{4}\right) \equiv\left(u_{1}, v_{1}, \mu_{1}, v_{1}\right)$, for parameters: $\mu_{j}=1-\alpha_{j+1}^{-1}, v_{j}=$ $\alpha_{1}^{-1}-\alpha_{j+1}^{-1}$. This integrable lattice is a discretization of the Boussinesq equation and its Bäcklund transformations. Solving these equations in terms of $\left(u_{1}, u_{2}\right)$ and $\left(v_{1}, v_{2}\right)$, we obtain the map

$$
\begin{aligned}
R(\boldsymbol{\mu}, \boldsymbol{v}):\left(\left(x_{1}, x_{2}\right),\left(y_{1}, y_{2}\right)\right) \mapsto & \left(\left(\frac{x_{1}\left(\mu_{2} x_{2}-v_{2} y_{2}\right)}{\mu_{1} x_{1}-v_{1} y_{1}}, \frac{\mu_{3} y_{1} y_{2}-v_{3} x_{1} x_{2}}{x_{1} y_{1} y_{2}\left(\mu_{2} x_{2}-v_{2} y_{2}\right)}\right),\right. \\
& \left.\left(\frac{y_{1}\left(\mu_{2} x_{2}-v_{2} y_{2}\right)}{\mu_{1} x_{1}-v_{1} y_{1}}, \frac{\mu_{3} y_{1} y_{2}-v_{3} x_{1} x_{2}}{x_{1} x_{2} y_{1}\left(\mu_{2} x_{2}-v_{2} y_{2}\right)}\right)\right),
\end{aligned}
$$

such that $R(\boldsymbol{\mu}, \boldsymbol{v})(\mathbf{u}, \mathbf{v})=\left(T_{m_{1}} \mathbf{u}, T_{\ell} \mathbf{v}\right)$. On the other hand, solving in terms of $\left(u_{1}, u_{2}\right)$ and $\left(T_{\ell} v_{1}, T_{\ell} v_{2}\right)$ we obtain the map $\widetilde{R}(\boldsymbol{\mu}, \boldsymbol{v}): \quad\left(\left(x_{1}, x_{2}\right),\left(y_{1}, y_{2}\right)\right) \mapsto$

$$
\begin{aligned}
& \left(\left(\frac{x_{1}\left(\mu_{2} \mu_{3} x_{1}^{-1}+\mu_{3} v_{1}\left(x_{1} x_{2}\right)^{-1} y_{1}+v_{1} v_{2} y_{1} y_{2}\right)}{\mu_{1} \mu_{3} x_{2}^{-1}+\mu_{1} v_{2} x_{1} y_{2}+v_{2} v_{3} y_{1}^{-1}}, \frac{x_{2}\left(\mu_{1} \mu_{3} x_{2}^{-1}+\mu_{1} v_{2} x_{1} y_{2}+v_{2} v_{3} y_{1}^{-1}\right)}{\mu_{1} \mu_{2} x_{1} x_{2}+\mu_{2} v_{3} x_{2}\left(y_{1} y_{2}\right)^{-1}+v_{1} v_{3} y_{2}^{-1}}\right),\right. \\
& \left.\left(\frac{y_{1}\left(\mu_{1} \mu_{3} x_{2}^{-1}+\mu_{1} v_{2} x_{1} y_{2}+v_{2} v_{3} y_{1}^{-1}\right)}{\mu_{2} \mu_{3} x_{1}^{-1}+\mu_{3} v_{1}\left(x_{1} x_{2}\right)^{-1} y_{1}+v_{1} v_{2} y_{1} y_{2}}, \frac{y_{2}\left(\mu_{1} \mu_{2} x_{1} x_{2}+\mu_{2} v_{3} x_{2}\left(y_{1} y_{2}\right)^{-1}+v_{1} v_{3} y_{2}^{-1}\right)}{\mu_{1} \mu_{3} x_{2}^{-1}+\mu_{1} v_{2} x_{1} y_{2}+v_{2} v_{3} y_{1}^{-1}}\right)\right) .
\end{aligned}
$$


The map $R$, taken together with $\widetilde{R}$, satisfies a 'twisted' YB equation,

$$
R_{12}\left(\lambda_{1}, \lambda_{2}\right) R_{13}\left(\lambda_{1}, \lambda_{3}\right) \widetilde{R}_{23}\left(\lambda_{2}, \lambda_{3}\right)=\widetilde{R}_{23}\left(\lambda_{2}, \lambda_{3}\right) R_{13}\left(\lambda_{1}, \lambda_{3}\right) R_{12}\left(\lambda_{1}, \lambda_{2}\right),
$$

which is equivalent to the $3 \mathrm{D}$ consistency condition for the lattice (50), as used in the context of quad-graphs [3]. However, the dual map $\widetilde{R}$ itself turns out to be a genuine YB map, and the YB equation it satisfies is again essentially equivalent to the $3 \mathrm{D}$ consistency for the lattice equations at hand. Note as well that both YB maps are indeed ultradiscretizable for a suitable choice of the parameters.

The lattice equations and corresponding YB maps obtained from the constraint (30) can be treated as a degenerate case of the present one. However, difficulties arise when one tries to interpret the lattice obtained from (29), and it is not clear if it corresponds to a YB map at all. Interestingly enough, the lattices that do correspond to YB maps bear no obvious relation to those discussed in [18] in relation to quadgraphs. These problems, as well as the study of the BBSs related to the above YB maps deserve further attention.

ACKNOWLEDGEMENTS. S. K. wishes to acknowledge support from the Japan Society for the Promotion of Science (JSPS) through a Grant-In-Aid for Scientific Research (no. 19540228); R. W. also acknowledges support by JSPS through a Grantin-Aid (no. 18540204).

\section{REFERENCES}

1. A. Berenstein and D. Kazhdan, Geometric and unipotent crystals, Geom. Funct. Anal., Special Volume, 'Part I' (2000), 188-236.

2. M. Białecki, On discrete Sato-like theory with some specializations for finite fields, RIMS kōkyūroku (to appear).

3. A. I. Bobenko and Y. B. Suris, Integrable systems on quad-graphs, Int. Math. Res. Not. 11 (2002), 573-611.

4. E. Date, M. Jimbo, M. Kashiwara and T. Miwa, Transformation groups for soliton equations: Euclidean Lie algebras and reduction of the KP hierarchy, Publ. RIMS, Kyoto Univ. 18 (1982), 1077-1110.

5. E. Date, M. Jimbo and T. Miwa, Method for generating discrete soliton equation. II, J. Phys. Soc. Jpn. 51 (1982), 4125-4131.

6. V. G. Drinfeld, On some unsolved problems in quantum group theory, in Lecture Notes in Mathematics, vol. 1510 (Kulish P. P., Editor) (Springer, Berlin, 1992), 1-8.

7. P. Etingof, Geometric Crystals and set-theoretical solutions to the quantum YangBaxter equation, Comm. Algeb. 31 (2003), 1961-1973.

8. G. Hatayama, K. Hikami, R. Inoue, A. Kuniba. T. Takagi and T. Tokihiro, The $A_{N}^{(1)}$ automata related to crystals of symmetric tensors, J. Math. Phys. 42 (2001), 274-308.

9. R. Hirota, Discrete analogue of a generalized Toda equation, J. Phys. Soc. Jpn. 50 (1981), 3785-3791.

10. A. Kuniba, M. Okado, R. Sakamoto, T. Takagi and Y. Yamada, Crystal interpretation of Kerov-Kirillov-Reshetikhin bijection, Nuclear Phys. B 740 (2006), $299-327$.

11. A. Kuniba, M. Okado, T. Takagi and Y. Yamada, Geometric crystal and tropical $R$ for $D_{n}^{(1)}$, Int. Math. Res. Not. 48 (2003), 2565-2620.

12. T. Miwa, On Hirota's difference equations, Proc. Jpn. Acad. Ser. A Math. Sci. 58(1982), $9-12$.

13. F. W. Nijhoff, The direct linearizing transform for the $\tau$ function in three-dimensional lattice equations, Phys. Lett. A 110 (1985), 10-14.

14. F. W. Nijhoff, Theory of integrable three-dimensional lattice equations, Lett. Math. Phys. 9 (1985), 235-241. 
15. F. W. Nijhoff, G. R. W. Quispel and H. W. Capel, Direct linearization of nonlinear difference-difference equations Phys. Lett. A 97 (1983), 125-128.

16. J. J. C. Nimmo, Darboux transformations and the discrete KP equation, J. Phys. $A \mathbf{3 0}$ (1997), 8693-8704.

17. Y. Ohta, R. Hirota, S. Tsujimoto and T. Imai, Casorati and Disrete gram type determinant representations of solutions to the discrete KP hierarchy, J. Phys. Soc. Jpn. 62 (1993), 1872-1886.

18. V. G. Papageorgiou, A. G. Tongas and A. P. Veselov, Yang-Baxter maps and symmetries of integrable equations on quad-graphs, J. Math. Phys. 47 (2006), 083502.

19. G. Post, Note on 'N-pseudoreductions' of the KP hierarchy, J. Phys. A 20 (1987), $6591-6592$.

20. D. Takahashi and J. Matsukidaira, Box and ball system with a carrier and ultradiscrete modified KdV equation, J. Phys. A 30 (1997), L733-L739.

21. D. Takahashi and J. Satsuma, A soliton cellular automaton, J. Phys. Soc. Jpn. 59 (1990), 3514-3519.

22. T. Tokihiro, D. Takahashi, J. Matsukidaira and J. Satsuma, From soliton equations to integrable cellular automata through a limiting procedure, Phys. Rev. Lett. 76 (1996), 3247-3250.

23. S. Tsujimoto, On a discrete analogue of the two-dimensional Toda lattice hierarchy, Publ. Res. Inst. Math. Sci. 38 (2002), 113-133.

24. S. Tsujimoto and R. Hirota, Ultradiscrete KdV equation, J. Phys. Soc. Jpn. 67 (1998), $1809-1810$.

25. K. Ueno and K. Takasaki, Toda lattice hierarchy, in Advanced Studies in Pure Mathematics, vol. 4 (Okamoto, K., Editor) (North-Holland, Amsterdam, 1984), 1-94.

26. A. P. Veselov, Yang-Baxter maps and integrable dynamics, Phys. Lett. A 314 (2003), 214-221.

27. A. P. Veselov, Yang-Baxter maps: dynamical point of view. Combinatorial Aspect of Integrable Systems, in MSJ Mem, vol. 17 (Mathematical Society of Japan, Tokyo, 2007), 145-167.

28. R. Willox, A direct linearization of the KP hierarchy and an initial value problem for tau functions, RIMS kōkyūroku 1170 (2000), 111-118. 